\title{
New approaches for the evaluation of renal vascular function in diabetes
}

\author{
G. Jerums • R. J. MacIsaac
}

Received: 28 April 2011 / Accepted: 24 May 2011 / Published online: 28 June 2011

(C) Springer-Verlag 2011

\begin{abstract}
In this issue of Diabetologia, two new approaches are described for the assessment of intra-renal blood flow in people with diabetes. The first approach used the technique of dynamic assessment of the resistance index (RI) in the renal interlobar arteries before and after administration of sublingual glyceryl trinitrate, and the second used MRI to assess total renal blood flow in relation to mean arterial pressure, thereby enabling direct measurement of overall renal RI. The results of the first study raise the possibility that dynamic evaluation of the intra-renal RI could be used as an early detector of vascular alterations in type 2 diabetes, before the onset of microalbuminuria. The results of the second study suggest that decreases in renal blood flow in people with longstanding type 1 diabetes reflect intra-renal vascular stiffening and raise the possibility that in microalbuminuric patients it may also reflect increased intraglomerular pressure.
\end{abstract}

Keywords Chronic kidney disease · Diabetes .

Nephropathy Magnetic resonance imaging .

Microalbuminuria $\cdot$ Renal resistance index

\section{Abbreviations \\ ACR Albumin to creatinine ratio}

\section{G. Jerums $(\bowtie)$}

Endocrine Centre, Austin Health \& University of Melbourne,

Heidelberg Repatriation Hospital,

Heidelberg West, PO Box 5444, VIC 3081, Australia

e-mail: ah-endo@unimelb.edu.au

\section{R. J. MacIsaac}

Department of Endocrinology and Diabetes,

St Vincent's Hospital \& University of Melbourne,

Fitzroy, VIC, Australia $\begin{array}{ll}\text { CKD } & \text { Chronic kidney disease } \\ \text { RI } & \text { Resistance index } \\ \text { RVR } & \text { Renal vascular resistance }\end{array}$

Non-invasive assessment of intra-renal blood flow has traditionally been performed using renal duplex Doppler ultrasound of the interlobar cortical arteries. The ratio of the difference between peak systolic and end diastolic velocity divided by peak systolic velocity, termed the intra-renal resistance index (RI), has provided an index of intra-renal arterial stiffness. In type 2 diabetes, intra-renal RI increases with age, blood pressure, BMI, the severity of markers of systemic atherosclerosis and left ventricular diastolic dysfunction, and decreased glomerular filtration rate (GFR) [1-3]. This association between increased intrarenal RI with decreased GFR remains after allowance for age, blood pressure and the degree of albuminuria [1]. Furthermore, higher baseline intra-renal RI measurements are associated with a greater subsequent decline in GFR [4]. There is also some evidence to suggest that the intra-renal RI differs depending on the underlying renal disease and is increased to a greater extent in people with diabetic renal diseases compared with non-diabetic renal diseases even after adjustment for multiple clinical variables, including creatinine clearance [5].

Two new approaches have recently been described for the assessment of intra-renal blood flow in people with diabetes. The first approach used the concept of dynamic assessment of RI in the renal interlobar arteries. This method has previously been used to assess intra-renal blood changes induced by the administration of renin-angiotensin system blocking agents [6]. However, in this instance, intrarenal RI was assessed by Bruno et al. [7] before and after administration of sublingual glyceryl trinitrate. In the second 
study, by Thelwall et al. [8], MRI was used to assess total renal blood flow in relation to mean arterial pressure, thereby enabling direct measurement of overall renal RI.

The study by Bruno et al. [7] involved 27 control participants, 49 participants with essential hypertension, and 32 participants with type 2 diabetes and normoalbuminuria. Ages were 51, 52 and 53 years and estimated GFRs were 89,92 and $87 \mathrm{ml} \mathrm{min}^{-1} 1.73 \mathrm{~m}^{-2}$ in the three study groups, respectively. The median albumin to creatinine ratio (ACR) in participants with type 2 diabetes was $0.88 \mathrm{mg} /$ mmol $(7.8 \mathrm{mg} / \mathrm{g})$, which was approximately fivefold greater than the median ACR for participants with essential hypertension or those in the control group, but was well below the accepted microalbuminuric range of $3-35 \mathrm{mg} /$ mmol $(30-300 \mathrm{mg} / \mathrm{g})$. The mean basal intra-renal RI was higher in diabetic $(0.65)$ than in hypertensive participants $(0.59, p<0.05)$ and controls $(0.58, p<0.05)$. However the expected decrease in RI induced by the administration of sublingual glyceryl trinitrate was greatest in controls $(11.1 \%)$, diminished in hypertensive participants $(9.0 \%, p<0.05)$ and further attenuated in participants with diabetes $(7.1 \%, p<0.05$ compared with hypertensive participants).

In the overall population, changes in RI induced by glyceryl trinitrate were independently related to serum glucose, reactive hyperaemia and aortic pulse wave velocity. In contrast, brachial artery response to glyceryl trinitrate was similar in the three study groups. Based on these results, the authors raise the possibility that dynamic evaluation of the intra-renal RI could be used as an early detector of vascular alterations in type 2 diabetes, before the onset of microalbuminuria.

The study by Thelwall et al. [8] was performed in ten control participants, nine normotensive participants with type 1 diabetes and normoalbuminuria, and eight participants with type 1 diabetes and microalbuminuria. Ages were 40, 40 and 43 years and estimated GFRs were $>60,83$ and $72 \mathrm{ml} \mathrm{min}^{-1} 1.73 \mathrm{~m}^{-2}$ in the three study groups, respectively. Mean duration of diabetes for the participants with type 1 diabetes was 27 and 28 years for the normoalbuminuria and microalbuminuric groups, respectively. All microalbuminuric participants were taking at least one renin-angiotensin system inhibitor with six also taking additional antihypertensive therapy. Renal MRI was used to assess renal perfusion, tissue oxygenation and kidney size in the three groups.

Mean renal artery flow (per single kidney) was lower in the whole diabetes cohort than in the control group (591 vs $\left.740 \mathrm{ml} \mathrm{m^{-1 }} 1.73 \mathrm{~m}^{-2}, p<0.05\right)$ when assessed by phase contrast angiography. Renal vascular resistance (RVR) was calculated as mean arterial pressure $(\mathrm{mmHg})$ divided by total renal blood flow $\left(\mathrm{ml} \mathrm{min}{ }^{-1} 1.73 \mathrm{~m}^{-2}\right.$ ). RVR increased from control to normoalbuminuric subgroup to microalbuminuric subgroup of type 1 diabetic people $(0.061 \mathrm{vs}$ 0.077 vs $0.093 \mathrm{~mm} \mathrm{Hg} \mathrm{ml}{ }^{-1} \times \min \times 1.73 \mathrm{~m}^{-2}, p=0.01$, respectively). RVR correlated inversely with estimated GFR in normo- but not in microalbuminuric people.

The authors suggest that decreases in renal blood flow in longstanding type 1 diabetes reflects intra-renal vascular stiffening and raise the possibility that in microalbuminuric patients it may also reflect increased intraglomerular pressure. However, since mean arterial pressure was $17 \%$ higher in the microalbuminuric than in the normoalbuminuric and control participants, this in itself could explain the increase in RVR in the microalbuminuric group.

Both of the above studies demonstrate that changes in renal blood flow are demonstrable in patients with type 2 or type 1 diabetes before the onset of microalbuminuria, as currently defined. Both studies demonstrate changes in renal blood vessel function independent of increases in albuminuria. However, small increases in ACR were already evident in the 'normoalbuminuric' individuals studied by Bruno et al. [7].

Both studies have certain limitations, in particular the small sample sizes. In addition, the lack of longitudinal data does not allow differentiation of cause, effect or association for the relationship of RI or RVR to the multiple clinical and vascular variables that were measured and included in the analyses. Furthermore, renal MRI remains a complex procedure that is not suited for large-scale application.

Despite these limitations, the above studies have potential clinical importance since normoalbuminuric renal insufficiency occurs in both type 1 [9-11] and type 2 diabetes [12-14], and may explain up to $25 \%$ of chronic kidney disease (CKD) in patients with type 2 diabetes [12, 13]. Normoalbuminuric renal insufficiency is not confined to diabetes and has also been described in large scale community studies [15].

The above studies raise the possibility that acute and long-term changes in blood vessels may contribute to the development of CKD in type 2 diabetes. Larger studies are needed in order to differentiate the separate roles of ischaemia, fibrosis, blood pressure, dyslipidaemia and glycaemic control in the evolution of CKD in both types of diabetes. Further dynamic renal RI studies that assess the effects of other endothelium-modulating agents, apart from glyceryl trinitrate, also need to be performed.

Acknowledgements Research in the authors' departments has been supported by grants from the National Health and Medical Research Council, The Austin Medical Research Foundation, the Diabetes Australia Research Trust and a Cardio-Vascular-Lipid research grant from Pfizer.

Both authors interpreted the data described in this commentary and contributed to the drafting and revising of the article. Both have approved the final version of the article.

Duality of interest The authors declare that there is no duality of interest associated with this manuscript. 


\section{References}

1. Taniwaki H, Nishizawa Y, Kawagishi T et al (1998) Decrease in glomerular filtration rate in Japanese patients with type 2 diabetes is linked to atherosclerosis. Diabetes Care 21:1848-1855

2. Macisaac R, Panagiotopoulos S, McNeil K et al (2006) Is nonalbuminuric renal insufficiency in type 2 diabetes related to an increase in intrarenal vascular disease? Diabetes Care 29:1560-1566

3. MacIsaac RJ, Thomas MC, Panagiotopoulos S et al (2008) Association between intrarenal arterial resistance and diastolic dysfunction in type 2 diabetes. Cardiovasc Diabetol 7:15

4. Nosadini R, Velussi M, Brocco E et al (2006) Increased renal arterial resistance predicts the course of renal function in type 2 diabetes with microalbuminuria. Diabetes 55:234-239

5. Ohta Y, Fujii K, Arima H et al (2005) Increased renal resistive index in atherosclerosis and diabetic nephropathy assessed by Doppler sonography. J Hypertens 23:1905-1911

6. Taniwaki H, Ishimura E, Kawagishi $T$ et al (2003) Intrarenal hemodynamic changes after captopril test in patients with type 2 diabetes: a duplex Doppler sonography study. Diabetes Care 26:132-137

7. Bruno RM, Daghini E, Landini L et al (2011) Dynamic evaluation of renal resistive index in normoalbuminuric patients with newly diagnosed hypertension or type 2 diabetes. Diabetologia. doi:10.1007/s00125-011-2148-y

8. Thelwall PE, Taylor R, Marshall SM (2011) Non-invasive investigation of kidney disease in type 1 diabetes by magnetic resonance imaging. Diabetologia. doi:10.1007/s00125-0112163-z

9. Caramori ML, Fioretto P, Mauer M (2003) Low glomerular filtration rate in normoalbuminuric type 1 diabetic patients: an indicator of more advanced glomerular lesions. Diabetes 52:10361040

10. Lane PH, Steffes MW, Mauer SM (1992) Glomerular structure in IDDM women with low glomerular filtration rate and normal urinary albumin excretion. Diabetes 41:581-586

11. Molitch ME, Steffes M, Sun W et al (2010) Development and progression of renal insufficiency with and without albuminuria in adults with type 1 diabetes in the Diabetes Control and Complications Trial and the Epidemiology of Diabetes Interventions and Complications Study. Diabetes Care 33:1536-1543

12. Kramer HJ, Nguyen QD, Curhan G, Hsu CY (2003) Renal insufficiency in the absence of albuminuria and retinopathy among adults with type 2 diabetes mellitus. JAMA 289:3273-3277

13. MacIsaac RJ, Tsalamandris C, Panagiotopoulos S, Smith TJ, McNeil KJ, Jerums G (2004) Nonalbuminuric renal insufficiency in type 2 diabetes. Diabetes Care 27:195-200

14. Tsalamandris C, Allen TJ, Gilbert RE et al (1994) Progressive decline in renal function in diabetic patients with and without albuminuria. Diabetes 43:649-655

15. Thomas MC, Macisaac RJ, Jerums G et al (2009) Nonalbuminuric renal impairment in type 2 diabetic patients and in the general population (National Evaluation of the Frequency of Renal impairment cO-existing with NIDDM [NEFRON] 11). Diabetes Care 32:1497-1502 